\title{
On Machine Measurement of Probe Error Research Based on Bayesian
}

\author{
Network \\ Liwen $\mathrm{Yan}^{\mathrm{a}}$, Yan $\mathrm{Yan}^{\mathrm{b}}$,Huaiqiang $\mathrm{Wu}^{\mathrm{c}}$ \\ Tianjin Key Laboratory of High Speed Cutting \&Precision Machining, Tianjin University of \\ Technology and Education, 300222 Tianjin, China \\ aylw618@163.com, ${ }^{b} x i u y u a n 009 @ s i n a . c o m,{ }^{c} 11$ wuhuaiqiang@163.com
}

Key words: Bayesian network, speed parameter, probe error, model

Abstract: Probe calibration is carried out under specific conditions; most of the error caused by the change of speed parameter has not been corrected, In order to reduce the measuring error influence on measurement accuracy, analyze the relationship between speed parameter and probe error, and using Bayesian network to establish the model of probe error. Model takes account of prior knowledge and sample data, with the updating of data, which can reflect the change of the errors of the probe and constantly revised modeling results.

\section{Introduction}

The probe is the key components of measurement system; its precision affects the overall measurement accuracy of system, which measures workpiece in the process and obtains the measurement data. According to the measurement results of treatment modify the process or compensate the machining error by other means, which improve machining precision of workpiece greatly and reduce defective index. The use of probe in this article is the British Renishaw OMP60 probe.

\section{Probe errors sources}

Using the probe to measure the workpiece, which usually with higher fast moving velocity close to the measured feature, reach approach distance, decelerate to measure velocity, measure the workpiece. The process of acceleration, deceleration and distance of acceleration and deceleration will affect trigger accuracy of probe system, which result the errors that directly affects the accuracy of the measurement data.

The measurement data obtained are the displacement of the probe $\left(l_{1}\right.$ or $\left.l_{2}\right)$ in the measurement, as shown in Fig.1 [1]. In order to obtain the measured size of the workpiece, the external dimensions subtract the diameter of the probe $\left(d_{0}\right)$, Instead of internal size add the diameter of the probe $\left(d_{0}\right)$. The total deformation of the measuring process are $f$, the actual amount of displacement of the probe then becomes $l_{1}^{\prime}$ or $l_{2}^{\prime}$, Therefore, the introduction of an effective role in the probe diameter concepts: $d=d_{0}-2 f$. In this case, the outer dimensions of the measurement: $L=l_{1}^{\prime}-d$; measuring the dimensions: $L=l_{2}^{\prime}+d$. The concept of effective role of diameter is to change the probe diameter [2]. 

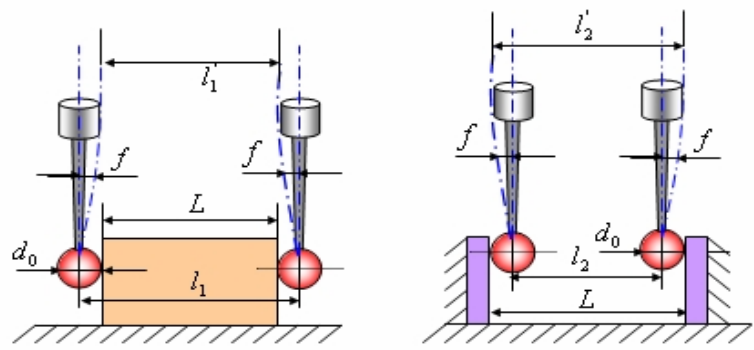

Fig.1 Size measurement

Without considering the influence of DCC (Direct Computer Control) parameters on the probe calibration, a large proportion of probe error is not fixed. DCC parameters: $V_{1}$ (fast moving velocity), $V_{2}$ (probing velocity), $D$ (approach distance). Therefore, we must consider DCC parameters to get the effective functional diameter of probe at different measuring parameters in the calibration and correction of probe. This article will combine different DCC parameters to analyze functional diameter of probe and on the probe error modeling research.

\section{Probe errors modeling research based on Bayesian Network}

Bayesian Network. Bayesian network contains the Bayesian network structure (directed acyclic graph) and Bayesian network parameters (conditional probability distribution table) [3].For $X=\left\{x_{1}, x_{2}, \ldots, x_{n}\right\}, x_{1}, x_{2}, \ldots, x_{n}$ corresponding to each node in a Bayesian network, the joint probability density $P\left(x_{1}, x_{2}, \ldots, x_{n}\right)$ is:

$$
\begin{aligned}
P\left(x_{N+1} \mid D, S^{h}\right)= & \int P\left(x_{N+1} \mid \theta_{s}, D, S^{h}\right) P\left(\theta_{s} \mid D, S^{h}\right) d \theta_{s} \\
& =\int P\left(x_{1} \mid \theta_{s}, D, S^{h}\right) P\left(x_{2} \mid x_{1}, \theta_{s}, D, S^{h}\right) \mathrm{L} \\
& P\left(x_{n} \mid x_{1}, \mathrm{~L}, x_{n-1}, \theta_{s}, D, S^{h}\right) P\left(\theta_{s} \mid D, S^{h}\right) \\
& =\int \prod_{i=1}^{n} \theta_{i j k} P\left(\theta_{s} \mid D, S^{h}\right) d \theta_{s} \\
& =E_{p\left(\theta_{s} \mid D, S^{h}\right)}\left(\prod_{k=1}^{r_{i}} \theta_{i j k}\right)=\prod_{i=1}^{n} \prod_{j=1}^{q_{i}} \frac{N_{i j k}^{\prime}+N_{i j k}}{N_{i j}^{\prime}+N_{i j}}
\end{aligned}
$$

$N_{i j}^{\prime}=\sum_{k=1}^{r_{i}} N_{i j k}^{\prime}, N_{i j}=\sum_{k=1}^{r_{i}} N_{i j k}$, parents $\left(\mathrm{x}_{\mathrm{i}}\right)$ for the father node $\mathrm{x}_{\mathrm{i}}$

Network node. The analysis of the probe error, it is the outcome of combined action of multiple factors. According to the previous to build variable set of model and parameters of variable domain: $V_{1}$ (fast moving velocity), $V_{2}$ (probing velocity), $D$ (approach distance). The variable set is $d=\left\{V_{1}, V_{2}, D\right\}$.

Build the network. Add the variables one by one into the figure one according to the order determined by causal relationship between variables. According to the conditional independence assumption, $d$ and DCC parameter has great relevance, so the parent node set is $\pi(x)=\left\{V_{1}, V_{2}, D\right\}$, 
no direct relationship between variables within the parent node. Each variable father node set add a directed edge point, get the probe error model of directed acyclic graph.

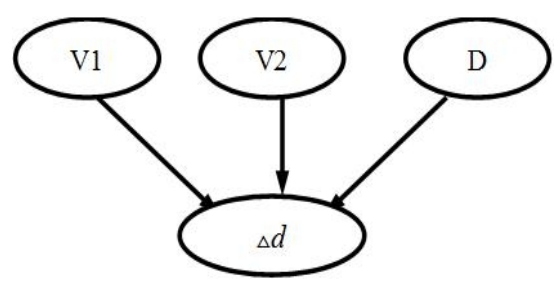

Fig.2 The probe errors network modeling

\section{Model verification}

Data acquisition. This experiment is in Dalian Machine Tool (FANUC0i-MD) to measure a nominal diameter of $25.0030 \mathrm{~mm}$ Standard ball, in different fast moving velocity, different probing velocity and the approach distance, to measure master ball and obtain experimental data. Try to keep the consistency of experimental conditions in the process of experiment, the Standard ball placed in the same fixed position, will other unnecessary factors as far as possible to minimize the influence on measurement accuracy.
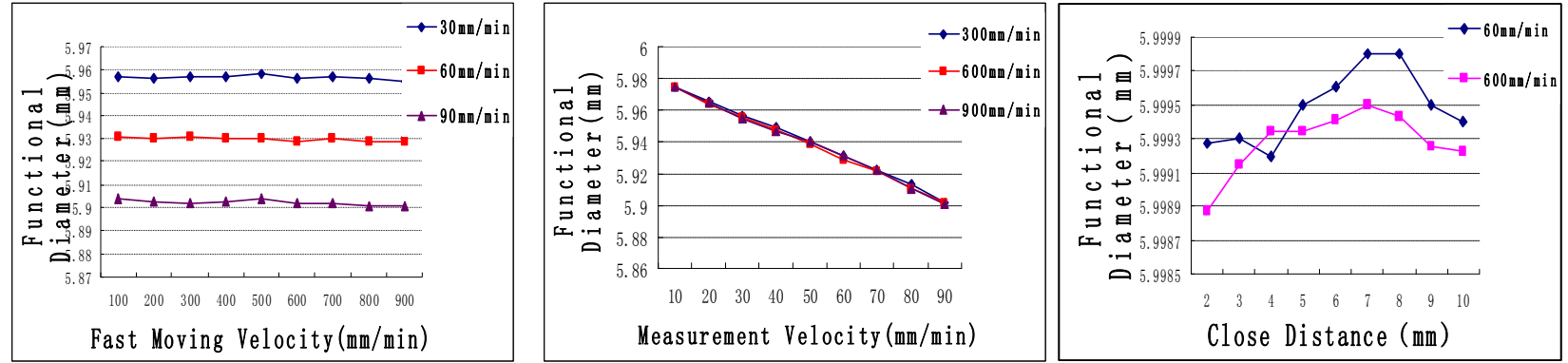

Fig.3 DCC parameters affecting the role of the diameter of the probe

In figure 1, functional diameter of probe and DCC parameters has greater relevance, especially measurement speed. Therefore, setting motion parameters and calibration motion parameters should be consistent in the actual measuring process, and the functional diameter of the probe should be calibrated before measuring.

Error prediction. When the probe error model is completed, according to the network and probability distribution, the joint probability distribution can be calculated based on maximum likelihood principle.

When the joint probability distribution is known, any node in the model can be calculated according Bayesian formula. For example, assuming a sampling results of the sampling points as follows: $V_{1}=a, V_{2}=b, D=\mathrm{c}$; the predictive probability of $\quad d$ as follows:

$$
\begin{aligned}
& p\left(d=i \mid V_{1}=a, V_{2}=b, D=\mathrm{c}\right)= \\
& \frac{p\left(V_{1}=a \mid d=i\right) p\left(V_{2}=b \mid d=i\right) p(D=\mathrm{c} \mid d=i)}{p\left(V_{1}=a\right) p\left(V_{2}=b\right) p(D=\mathrm{c})} * p(d=i)
\end{aligned}
$$

Using the test data to predict the results, according to the formula (4), we use 18 set of test data to make predictions, the statistical analysis shows that the average relative error is $7.2 \%$, Prediction accuracy is $83.3 \%$.table 1 shows the partial model prediction of $d$. 
Table 1 Probe error modeling results

\begin{tabular}{|c|l|l|l|l|l|l|l|l|l|l|l|l|l|l|}
\hline Sampling points & - & 5 & 6 & 7 & 8 & 9 & 10 & 11 & 12 & 13 & 14 & 15 & 16 & - \\
\hline$d$ statue value & - & 3 & 4 & 5 & 4 & 3 & 3 & 4 & 3 & 4 & 3 & 3 & 3 & - \\
\hline Predictive value & - & 3 & 4 & 3 & 4 & 3 & 3 & 4 & 3 & 2 & 3 & 3 & 3 & - \\
\hline Relative error $(\%)$ & - & 0 & 0 & 40 & 0 & 0 & 0 & 0 & 0 & 50 & 0 & 0 & 0 & - \\
\hline Success or not & - & $\mathrm{Y}$ & $\mathrm{Y}$ & $\mathrm{N}$ & $\mathrm{Y}$ & $\mathrm{Y}$ & $\mathrm{Y}$ & $\mathrm{Y}$ & $\mathrm{Y}$ & $\mathrm{N}$ & $\mathrm{Y}$ & $\mathrm{Y}$ & $\mathrm{Y}$ & - \\
\hline
\end{tabular}

Experimental analysis. In order to verify the validity of the intuitively, design the following experiment: Measurement of the standard ball.

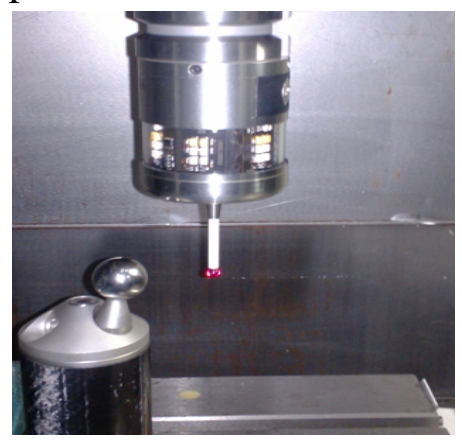

Fig.4 Standard ball experiments
Table 2 Experimental results

\begin{tabular}{|c|l|}
\hline Standard Ball Diameter: & $25.0030 \mathrm{~mm}$ \\
\hline Before the Error Compensation: & $24.978 \mathrm{~mm}$ \\
\hline After the Error Compensation: & $24.989 \mathrm{~mm}$ \\
\hline
\end{tabular}

\section{Conclusion}

Modeling method based on Bayesian network is different from the traditional fitting model, starting from the probability distribution of the data, on the one hand, using the language of graph theory visual expression to produce probe error causal dependencies between various factors, on the other hand, according to the principle of probability theory to analyze the inner relation between various factors, utilization, reduce the computational complexity of reasoning forecast, finally according to the target variable probability distribution prediction results are obtained, and modeling method is intuitive and high precision.

\section{Acknowledgments}

The work was supported by the Innovation Team Training Plan of Tianjin Universities and colleges (Grant No. TD12-5043), Tianjin Key Laboratory of High Speed Cutting \&Precision Machining and pre-research project of Tianjin University of technology and education (KJY15-01). The authors would like to thank the anonymous reviewers for their valuable comments.

\section{References}

[1] Ytai Fei, Jing Zhao: Coordinate Measuring Machines dynamic characteristics and dynamic accuracy study. The fourth cross strait Manufacturing Technology Symposium, (2004)

[2] Xiushui Ma: Research on Dynamic Error Source Analysis Modeling and Correction Technical of Coordinate Measuring Machines. HeFei University of Technology, (2005)

[3] Shuangcheng Wang: Learning, inference and application of Bayesian Network. Lixin Accounting Publisher, (2010) 